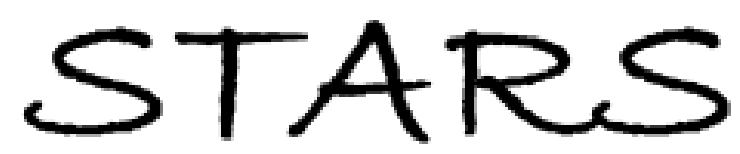

University of Central Florida

STARS

$1-1-2000$

\title{
All-fiber connectorized compact fiber optic delay-line modules using three-dimensional polarization optics
}

Nicholas Madamopoulos

Nabeel A. Riza

University of Central Florida

Find similar works at: https://stars.library.ucf.edu/facultybib2000 University of Central Florida Libraries http://library.ucf.edu

This Article is brought to you for free and open access by the Faculty Bibliography at STARS. It has been accepted for inclusion in Faculty Bibliography 2000s by an authorized administrator of STARS. For more information, please contactSTARS@ucf.edu.

\section{Recommended Citation}

Madamopoulos, Nicholas and Riza, Nabeel A., "All-fiber connectorized compact fiber optic delay-line modules using three-dimensional polarization optics" (2000). Faculty Bibliography 2000s. 2685.

https://stars.library.ucf.edu/facultybib2000/2685

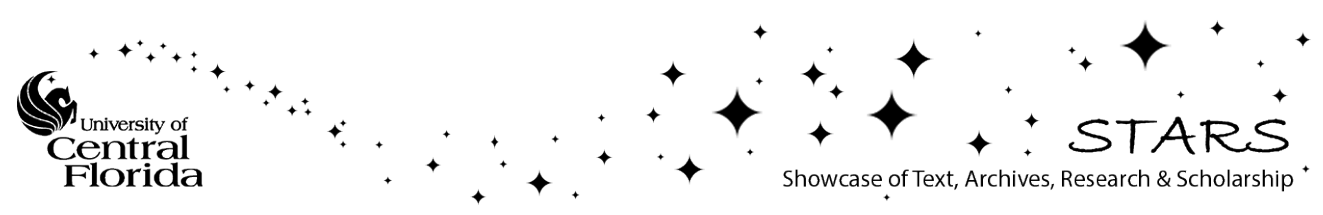




\section{All-fiber connectorized compact fiber optic delay-line modules using three-dimensional polarization optics}

\author{
Nicholas Madamopoulos \\ Nabeel A. Riza, FELLOW SPIE \\ Photonic Information Processing Systems \\ (PIPS) Laboratory \\ School of Optics/Center for Research and \\ Education in Optics and Lasers \\ University of Central Florida \\ P.O. Box 162700 \\ Orlando, Florida 32816-2700 \\ E-mail: riza@creol.ucf.edu
}

\begin{abstract}
Compact and all-fiber connectorized photonic delay-line modules based on three-dimensional bulk polarization optics are proposed and experimentally demonstrated. The modules are built on a single optical microbench and demonstrate optical leakage noise performance of $-40 \mathrm{~dB}$ at switching speeds of $10 \mu \mathrm{s}$. Insertion loss analysis is also performed. A special gradient-index lens fiber optic collimator design is proposed to further reduce the optical insertion loss of the delay-line module. A wavelength-dependent design is also proposed for expanding the applicability of the PDL module to multichannel operation. () 2000 Society of Photo-Optical Instrumentation Engineers. [S0091-3286(00)00809-6]
\end{abstract}

Subject terms: photonic time delay; ferroelectric; phased array antenna; fiber optic applications; polarization.

Paper 990396 received Oct. 10, 1999; revised manuscript received Feb. 21, 2000; accepted for publication Mar. 8, 2000.

\section{Introduction}

Reversible rapidly variable photonic delay lines (PDLs) are a powerful and important tool for many photonic information-processing applications. One key application is true-time-delay steering for phased array antennas. ${ }^{1}$ More recently, PDL applications have spread to other fields such as laser radars, ${ }^{2}$ ultrasound, ${ }^{3}$ optical memories, ${ }^{4}$ and astronomy. ${ }^{5}$

During the past few years we have concentrated our work in the implementation of $N$-bit multichannel PDL systems using electrically controllable polarization switching devices, such as millisecond-response-time nematic liquid crystal (NLC) devices ${ }^{6}$ and microsecond-response-time ferroelectric liquid crystal (FLC) devices. ${ }^{7,8}$ Nevertheless, these early bulk-optic and free-space-solid-optic systems were rather large in size and required careful alignment and fabrication of high-quality large-area multichannel NLC and FLC devices. ${ }^{9,10}$ Hence, these systems are appropriate for applications where overall system size and weight are not important.

Compact PDL modules are necessary for fielded systems where space and volume on the application platform (e.g., satellite) are limited. Thus, an important issue for the implementation of PDLs that use two-dimensional (2-D) polarization switches is packaging. So far, no ultracompact PDL design based on polarization bulk optics has been proposed. In addition, no polarization-based fiber PDL has been proposed that is easily adaptable to generate any userdesired optical delay. Current fiber optic (FO) cable and connector technology is an excellent candidate for interconnecting environmentally robust and well-engineered delay control modules. In this paper, a PDL module that meets the above-mentioned requirements, i.e., compactness and time-delay adaptability, is proposed and demonstrated. Our approach for achieving this PDL involves the use of micro- lens FO collimators and standard FC/PC (or FC/APC for reduced backreflection) connectorized fiber optics. Two different FO-collimator technologies are tested. The first one is based on spherical-microlens fiber ports, and the second one is based on gradient-index (GRIN) lens collimators. A compact single micro-optical-bench PDL is experimentally demonstrated for each of these technologies. ${ }^{11,12}$

\section{Alternative Compact PDL Module Designs}

A fiber connectorized $N$-bit PDL approach offers ease in assembly of $N$ bits, ease in maintenance and repair, and compact size via fiber delay paths. A typical fiber-optically interconnected PDL system can be formed by a cascade of single-bit compact PDLs (Fig. 1). This modular approach gives the flexibility to the system designer to choose from a wide range of available time delays and their combinations.

A typical fiber-optically connectorized PDL module can be implemented via a $1: 2$ FO switch that routes the signal to either the delay or the nondelay path, and a FO combiner (or 2:1 FO switch) that combines the two independent channels into one before the signal propagates to the next bit. Possible approaches to a compact PDL module include electromechanical FO switches and integrated electro-optic switches. Two possible PDL configurations based on the above switching technologies are shown in Fig. 2. The first technology can provide very good optical isolation (e.g., 60 $\mathrm{dB})$ and low insertion loss $(\approx 0.5 \mathrm{~dB}$ per switch, or $1.5 \mathrm{~dB}$ for the PDL), but rather slow switching speeds of $>7 \mathrm{~ms}{ }^{13}$ On the other hand, the integrated electro-optic switches offer faster switching speeds (e.g., 100 ps), but they have poor optical isolation of $\approx 22 \mathrm{~dB}$, and inherently high insertion loss $(\approx 4 \mathrm{~dB}) .{ }^{14}$ This high loss, in conjunction with the $\approx 3.5-\mathrm{dB}$ loss of a $2: 1 \mathrm{FO}$ combiner, leads to a PDL module with insertion loss of $>7.5 \mathrm{~dB}$ for both PDL settings. Our compact PDL module using bulk polarization 


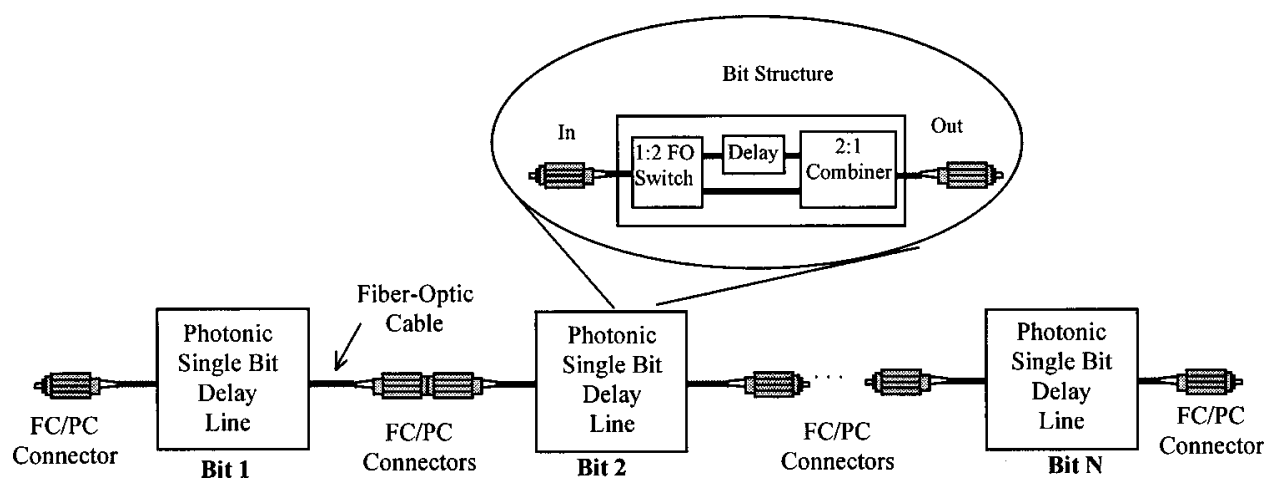

Fig. 1 A fiber-optically interconnected PDL system using a cascade of single-bit compact PDL modules.

optics and FLC polarization switches provides a system trade-off with $10-\mu$ s switching speeds, $>42-\mathrm{dB}$ optical isolation, ${ }^{15}$ and an expected loss of $<5.4 \mathrm{~dB}$.

\section{The Bulk-Optics Ferroelectric Liquid Crystal Based Compact PDL Module}

Figure 3 shows the proposed bulk-optics FLC-based PDL module. Polarization-maintaining fibers (PMFs) must be used at the input and output ports, since the module is based on polarization switching. On the other hand, a nonPMF is used at the delay path. The PDL is based on a reflective design that allows the use of Faraday rotatormirror (FRM) connectorized single-mode fiber (SMF) in the delay path with no signal degradation. ${ }^{16}$ Thus, shorter fiber lengths are required. Note that using FC/PC connectors for ease in (dis)connection allows the user to achieve

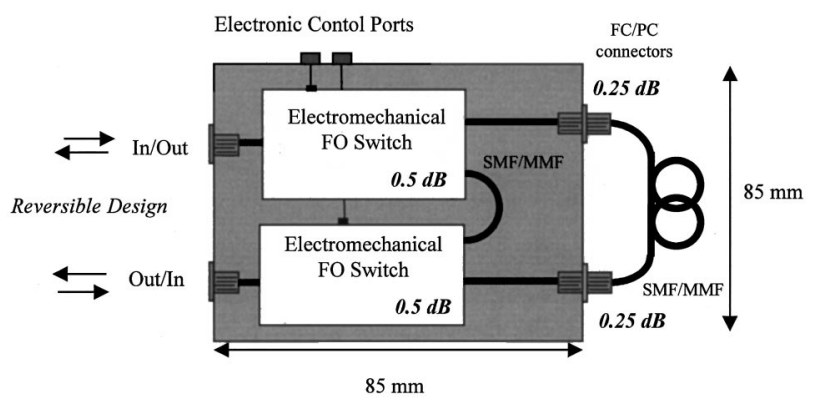

(a)

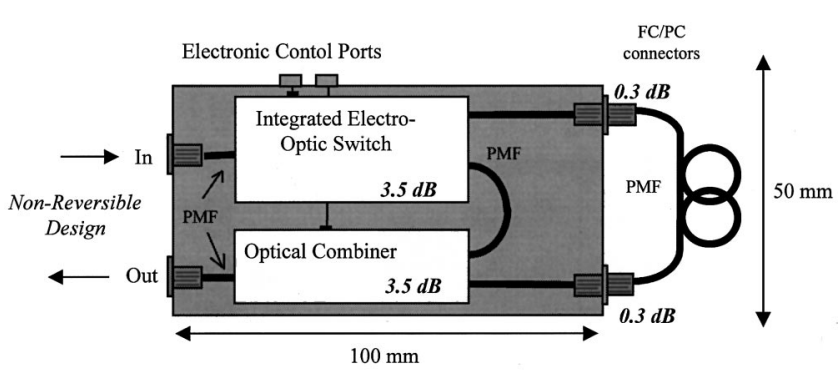

(b)

Fig. 2 (a) Electromechanical PDL, and (b) integrated electro-opticswitch PDL. (SMF: single-mode fiber; MMF: multimode fiber; PMF: polarization-maintaining fiber.) different time delays by simply changing the SMF lengths. The PDL module using fiber optic microlenses is expected to have optical losses of $3.2 \mathrm{~dB}$ for the nondelay path, and $5.4 \mathrm{~dB}$ for the delay path. The higher insertion loss for the delay path is due to the insertion loss of the FRM and the additional FC/PC connectors. These estimates are based on optical-component loss numbers given by the manufacturers and expected FLC device losses of $0.45 \mathrm{~dB}$ (or 10\%).

The following sections describe two experimental demonstrations of the switched PDL. The first one uses spherical microlenses, and the second one uses GRIN-lens FO collimators.

\section{Switched Compact PDL Based on Spherical- Microlens Fiber-Optic Collimators: Experimental Demonstration}

The experimental setup of the bulk-optics FLC-based switching fabric is shown in Fig. 4. An externally modulated FO link with a diode-pumped Nd:YAG laser at 1319 $\mathrm{nm}$ and a Mach-Zehnder electro-optic modulator is used. The modulated light is coupled into the PDL via a Fujikura PANDA-type PMF that can be used at 1319-nm wavelength. This light is collimated using a microlens positioned in an OFR fiber port (PAF-X), which has three translation $(x, y, z)$ and two tilt microcontrols. ${ }^{17} \mathrm{~A}$ linear polarizer is attached at the input port and is set to let only horizontally polarized ( $p$-polarized) light into the system. FLC devices are used as polarization switches (PSs). PS1 acts as a

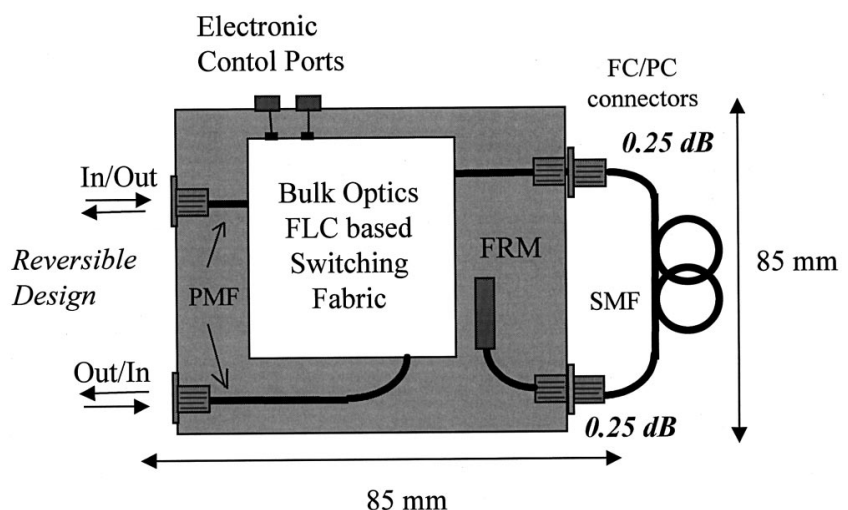

Fig. 3 The reversible bulk-optics FLC-based compact PDL module. 


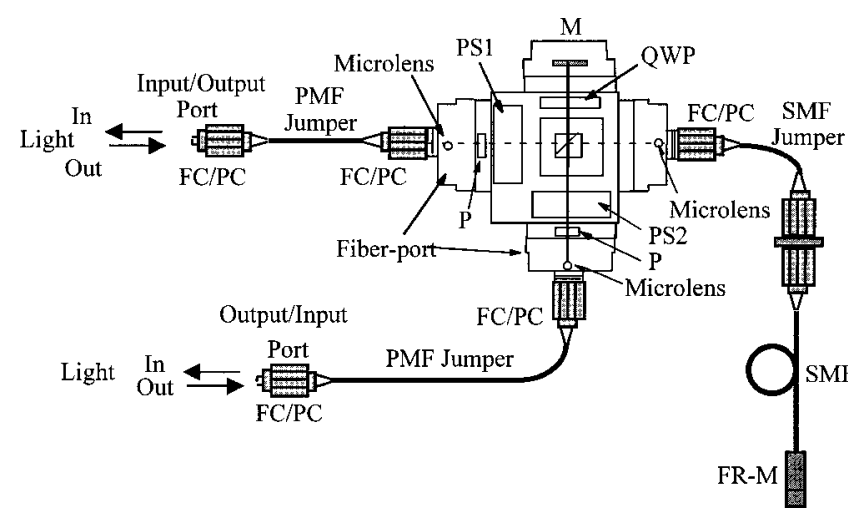

Fig. 4 The experimental setup of the compact PDL module based on spherical-microlens fiber ports.

switch to change the polarization to vertical (s polarization) when it is set in its on state, or leave the input polarization unchanged if it is set in the off state. When the light is $p$-polarized, it passes through the polarization beamsplitter cube (PBS) and follows the fiber delay path. On the other hand, when PS1 is set in its off state, the $s$-polarized light is deflected from the PBS and travels through the nondelay path. The polarization of the light rotates by $90 \mathrm{deg}$ after propagation through either of the two paths, and is directed through the PBS towards the output port. For example, this happens when vertically polarized light is deflected by 90 deg from the PBS. PS2 and an additional polarizer form the active noise filter to suppress any leakage noise coming from PS1 or the PBS. ${ }^{15}$ Output light is coupled via the PMF jumper to the photoreceiver. The delay path consists of a FC/PC-connectorized 10-cm-length SMF connected with a FC/PC-connectorized 3-m-long SMF terminated with a FRM. The SMF is a Corning SMF-28 that can be used at 1319-nm wavelength.

Figure 5 shows photographs of our compact PDL module built in the laboratory. The compact PDL was built on a $4 \times 4-\mathrm{cm}$ aluminum optical bench. The OFR fiber ports were mounted at its sides. The overall size of the compact $\mathrm{PDL}$, including the FC/PC connectors attached on the fiber ports, is $8 \times 8 \mathrm{~cm}$.

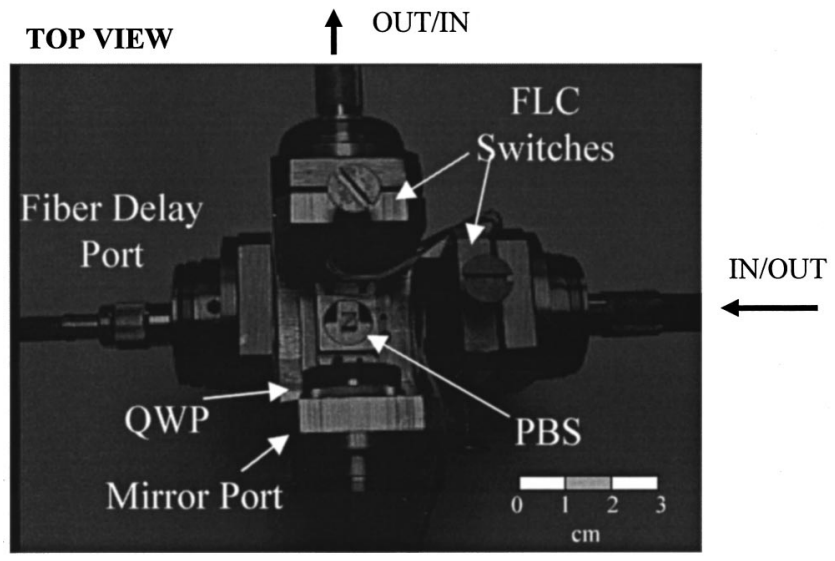

Fig. 5 Photograph of the compact photonic delay line based on spherical microlens fiber ports.
Table 1 Expected and measured optical insertion loss.

\begin{tabular}{lcccccc}
\hline \hline & \multicolumn{2}{c}{ Micro-optic-based PDL } & & \multicolumn{2}{c}{ GRIN-lens-based PDL } \\
\cline { 2 - 3 } PDL setting & $\begin{array}{c}\text { Expected } \\
\text { optical loss } \\
(\mathrm{dB})\end{array}$ & $\begin{array}{c}\text { Measured } \\
\text { optical loss } \\
(\mathrm{dB})\end{array}$ & & $\begin{array}{c}\text { Expected } \\
\text { optical loss } \\
(\mathrm{dB})\end{array}$ & $\begin{array}{c}\text { Measured } \\
\text { optical loss } \\
(\mathrm{dB})\end{array}$ \\
\hline Delay & 4.9 & 6.2 & & 5.6 & 6.9 \\
Nondelay & 3.1 & 4.6 & & 4.6 & 5.7 \\
\hline \hline
\end{tabular}

Table 1 shows the measured optical losses for the two settings as well as the optimum projected loss. The expected losses for our experimental PDL have been calculated from the optical-component losses given by the manufacturer and FLC insertion losses of $0.45 \mathrm{~dB}$ (or 10\%). The difference between the optimum loss and the experimentally measured data is due to the higher insertion loss of the Displaytech (Longmont, CO) FLC devices-viz., 0.96 and $0.75 \mathrm{~dB}(20 \%$ and $16 \%)$ - used in our experiment. This higher FLC-device loss leads to a 0.7-dB-higher insertion loss for our prototype. Additional losses $(\approx 0.7 \mathrm{~dB})$ are due to our imperfect alignment. In our experiments a $1319-\mathrm{nm}$ direct-modulated semiconductor laser was used as the source.

So far, researchers have used costly and sensitive microwave-band test instrumentation to characterize the time delays obtained from PDLs designed for radar and antenna control applications. As we show perhaps for the first time, time-delay measurements on a PDL can also be obtained using a low-frequency technique that does not require costly microwave test equipment. ${ }^{18}$ Time-delay measurements on our PDL were also obtained using this lowfrequency technique. For the nanosecond time delays required in radar applications, high-frequency signal generators, oscilloscopes, and network analyzers are required for accurate measurement of the time delays. In our simpler approach, a low-frequency square-wave generator can be used to drive the laser source. In this case, the light is modulated with the square pulse, and the delays can be detected using a low-frequency oscilloscope by tracking the onset or the offset of the square pulses instead of the very high (gigahertz) frequencies used in phased array radar applications. Figure 6 shows the low-frequency square-wave signals that are used to drive the microwave analog intensity modulator (top traces). Figure 6 also shows the photodetected signal at the output of the PDL module for both of its settings (bottom trace). We can conclude that the obtained time delay is $54.90 \mathrm{~ns}-23.90 \mathrm{~ns}=31 \mathrm{~ns}$, which is in agreement with the expected time delay from our $3.1-\mathrm{m}$ SMF delay.

The optical leakage noise was also measured for our compact PDL module. The optical leakage noise is defined as $10 \times \log [($ leakage-noise optical power)/(signal optical power)]. We define the signal power as the optical power in the beam of the desired polarization that travels through the desired delay or nondelay path of the module. All other optical power measured at the output is regarded as leakage noise optical power. For the nondelay setting the optical leakage noise was measured at $-39.7 \mathrm{~dB}$, while for the delay setting it was $-38.0 \mathrm{~dB}$. The higher leakage noise observed for the delay path is due to the lower insertion 


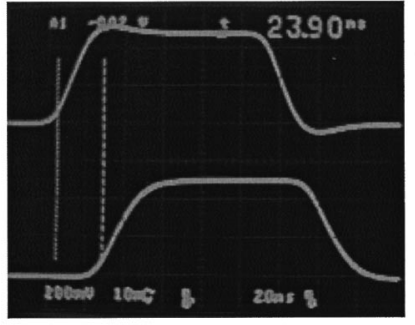

(a)

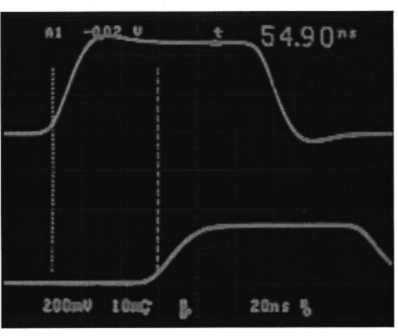

(b)
Fig. 6 Oscilloscope traces showing (a) the nondelayed and (b) the delayed signal. Top traces: signal driving the external modulator; bottom traces: photodetected signal at the output of the PDL module. The markers have been positioned at the onset of the pulse, where the pulse attains $10 \%$ of its maximum value.

loss of the nondelay path, which allows higher leakage noise to reach the output of the PDL than for the delay setting. However, the $-38-\mathrm{dB}$ leakage noise is highly desirable for phased array antenna applications.

\section{Switched Compact PDL Experimental Setup Based on GRIN-Lens Fiber Optic Collimators}

The design of a PDL module based on a GRIN-lens FO collimator is similar to the one presented in the previous section except that the spherical-microlens fiber ports are replaced by the GRIN-lens FO collimators. Light from an externally modulated FO link is fed via a PMF into the PDL. This light is collimated using a GRIN-lens FO collimator from OZ-Optics. The collimator is positioned in an OZ-Optics fiber collimator flange for tilt microcontrols ${ }^{19}$ and on a standard $x-y$ translation micropositioner from OptoSigma Corporation. ${ }^{20} \mathrm{~A}$ linear polarizer is attached at the input port and is set to let only horizontally polarized light into the PDL. FLC devices are used as PSs that either rotate the incident polarization by $90 \mathrm{deg}$ when they are set on, or leave the input polarization unchanged when they are set off. Depending on the polarization, the light can follow either the nondelay path or the delay (fiber) path. The output GRIN-lens FO collimator collects the optical signal and delivers it to the photoreceiver.

The optical loss for the two settings of our PDL was measured at 6.9 and $5.7 \mathrm{~dB}$ for the delay and nondelay paths, respectively. The expected optical loss for the delay path is $5.6 \mathrm{~dB}$, while that for the nondelay path is $4.6 \mathrm{~dB}$. This expected loss has been calculated from the opticalcomponent losses given by the manufacturers, the FLC insertion losses of $0.45 \mathrm{~dB}$ (or 10\%), and the expected FO coupling efficiency using GRIN-to-GRIN distances compatible with our PDL. Additional losses $(\approx 0.5 \mathrm{~dB})$ are due to imperfect alignment. Again the insertion loss of our PDL is mainly due to the triple-cell Displaytech, Inc., FLC devices and the limited coupling efficiency of the GRIN-lens FO collimator. The optical insertion loss of these PDLs can be improved by $\approx 0.9 \mathrm{~dB}$ if single-cell FLC devices with higher-birefringence FLC material (e.g., >0.12) are used. Single-cell, lower-insertion-loss (e.g., $0.3 \mathrm{~dB}$ ) FLC devices have recently been fabricated by Boulder Nonlinear Sys-

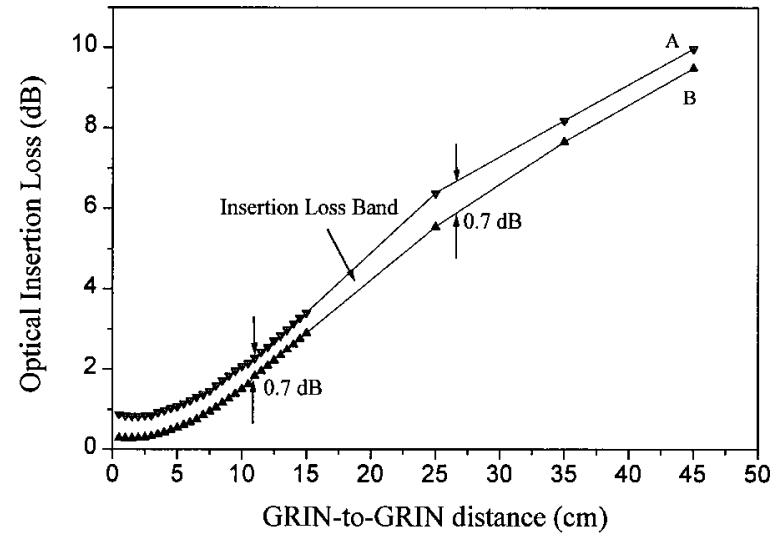

(a)

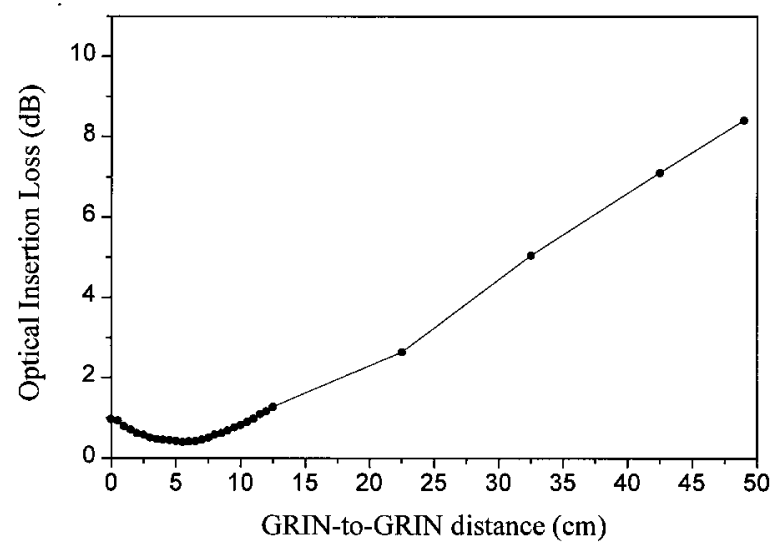

(b)

Fig. 7 Measured optical insertion losses for (a) pairs of $<0.25$-pitch GRIN-lens FO collimators as a function of their distance from each other (these collimators are designed to have the beam waist at the exit surface of the GRIN lens); (b) optimized coupling efficiency for larger GRIN-to-GRIN distances (e.g., $5.5 \mathrm{~cm}$ ) using specially designed $<0.25$-pitch GRIN-lens FO collimator with the beam waist not at the exit surface of the GRIN lens.

tems, Inc., Lafayette, $\mathrm{CO}$, and these devices have been used as PS devices in our 7-bit PDL system. ${ }^{10}$ Thus, using these lower insertion losses, the total module insertion loss for the delay and nondelay settings can be reduced to 6.0 and $4.8 \mathrm{~dB}$, respectively.

The insertion loss can also be improved if the coupling efficiency of the GRIN-lens FO-collimator assemblies can be improved. Although GRIN-lens collimator technology is rather mature, there are tolerances related to the fabrication of the fiber and its alignment with the GRIN-lens interconnects that limit the coupling efficiency, especially at separations of $>5 \mathrm{~cm}$. We have experimentally found that these loss numbers are highly dependent on the specific pair of fiber collimators used for the experiment. Experiments have shown that maximum coupling efficiency varies with different collimators and for each pair occurs at a different GRIN-to-GRIN separation. Figure 7(a) shows the experimental data for the optical insertion loss versus the GRINto-GRIN distance. This optical insertion loss can be found within a band $\approx 0.7 \mathrm{~dB}$ wide. Note that the beam propa- 


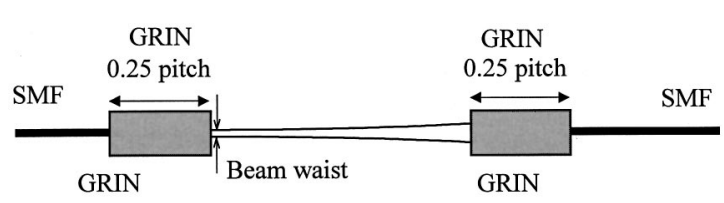

(a)

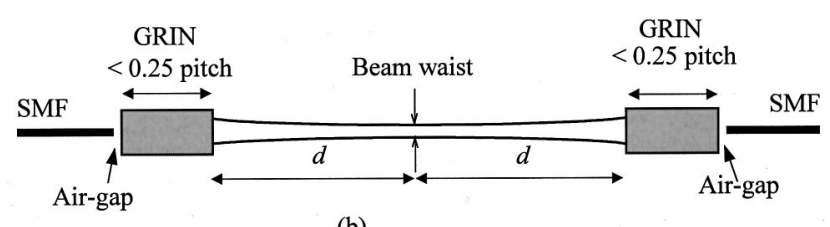

(b)

Fig. 8 (a) 0.25-pitch GRIN lens; (b) <0.25-pitch GRIN lens with an air gap between the lens and the fiber.

gates as a Gaussian beam with a specific beam divergence, thus expanding as it propagates in free space. A GRIN-lens FO collimator typically requires a 0.25 -pitch GRIN lens. Often a smaller-length (e.g., 0.23-pitch) GRIN lens is used, leaving an air gap between the lens and the fiber, in order to reduce the backreflection. ${ }^{21}$ This air gap can also be adjusted to set the beam waist at a specific distance from the face of the GRIN lens ${ }^{19}$ as shown in Fig. 8. This can result in a maximum coupling efficiency for a larger GRIN-toGRIN separation. The plots of Fig. 7(a) correspond to the maximum and minimum insertion losses for a range of different GRIN-lens FO collimators using a $<0.25$-pitch GRIN lens for reduced backreflection. The position of the fiber with respect to the GRIN lens has been adjusted so that the beam waist is at the exit surface of the lens. If the fiber is adjusted so that the beam waist is further away from the exit surface of the lens, maximum coupling efficiency is obtained for larger GRIN-to-GRIN distances. The experimental data in Fig. 7(b) show that the best coupling efficiency occurs for a 5.5-cm GRIN-to-GRIN distance. Thus, if two similarly fabricated $<0.25$-pitch GRIN-lens FO collimators with their waists at a distance $d$ from the lens are used, maximum or optimized coupling efficiency will be obtained for a GRIN-to-GRIN separation of $2 d$ [Fig. 8(b)]. Hence, it is possible to design and manufacture GRIN-lens FO collimators optimized for the free-space propagation of our PDL paths, leading to improved insertion losses for our PDL designs.

\section{Multichannel Operation of the Compact PDL Module}

The proposed single-physical-channel PDL module can be combined with wavelength-multiplexing techniques to obtain multichannel operation. This can be accomplished if a PMF with Bragg gratings is used in the fiber delay path. ${ }^{12}$

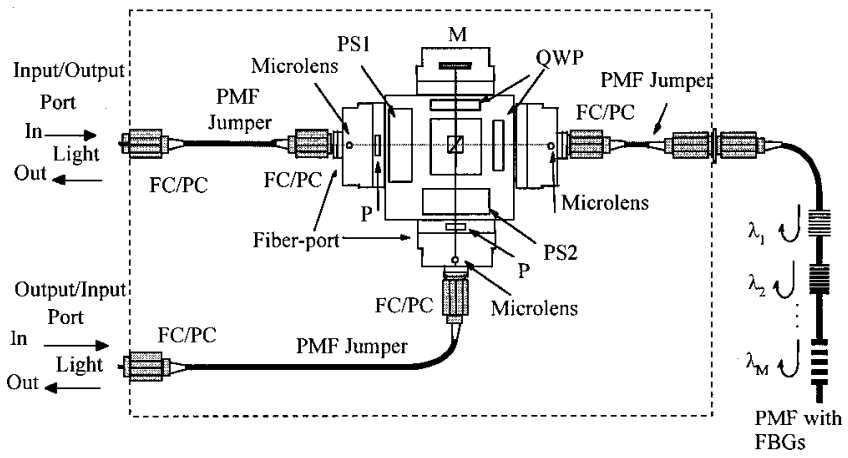

Fig. 9 Single-physical-channel, wavelength-dependent, compact PDL for multichannel operation using FBGs. (P: polarizer; M: mirror; PMF: polarization-maintaining fiber; QWP: quarter-wave plate; FBG: fiber Bragg grating; PS: polarization switch.)

In this PDL version, a quarter-wave plate (QWP) needs to be used in the fiber delay path. The QWP is placed in the fiber delay path so that there is a 90 deg rotation of the polarization of the light as it propagates through the QWP, the fiber, and the QWP again. Figure 9 shows such a design. The fiber Bragg gratings (FBGs) have to be recorded at specific distances from each other such that the desired time delays are obtained for the different wavelengths.

An alternative approach for the delay path consists of long PMFs (with no FBGs) and short PMFs with FBGs (Fig. 10). All the short PMFs are of the same length. Hence, the desired time delays can be obtained with appropriate choice of the long PMFs. This approach gives greater time-delay flexibility to the end user, since the time delays depend mainly on the length of the PMFs. A typical dimension of a PMF-pigtailed FBG is 15 to $20 \mathrm{~cm}$ from each side. ${ }^{22}$ The grating size is $\approx 5 \mathrm{~mm}$ and depends on the specification of the wavelength, the FWHM bandwidth, and the desired reflectivity.

Important issues with FBG-based fiber delay are the interchannel isolation and the effect of the distributed reflection of wavelengths from the FBGs. The interchannel isolation depends on the reflectivity of each FBG for the desired wavelength and on its transmission characteristics for the wavelengths outside its FWHM bandwidth. The distributed reflection happens because the FBG is not a thin interface mirror, but rather a 3-D structure, and it arises from constructive or destructive multiple-beam interference. Nevertheless, it is expected that the distributed reflection will not cause significant time jitter errors, in view of the small size of the FBGs. The worst case will be when part of the light incident on the FBG is reflected from the front end of the grating while the rest of it is reflected from the back end of the FBG. Thus a $10-\mathrm{mm}(2 \times 5 \mathrm{~mm})$ pathlength difference exists between the two portions of the

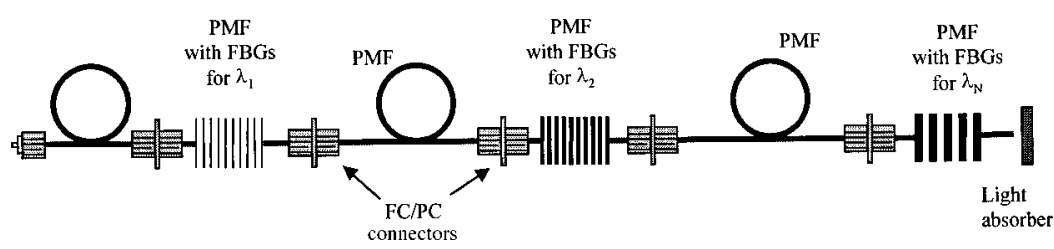

Fig. 10 Delay-path option based on alternating long PMFs without FBGs and short PMFs with FBGs. 


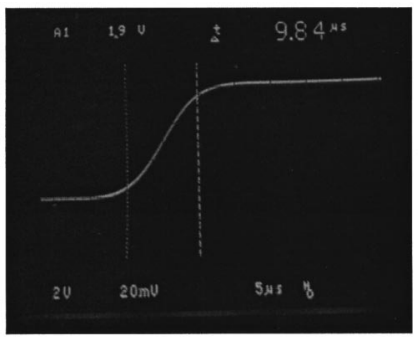

(a)

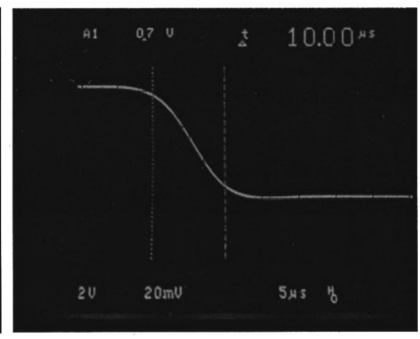

(b)
Fig. 11 FLC switching response at $1319 \mathrm{~nm}$ : (a) a 9.84- $\mu$ s rise time and (b) a $10-\mu$ s fall time.

light. Hence, a time jitter of $<20$ ps is expected.

This single-channel wavelength-dependent PDL approach can also be used in large photonic control systems for phased array antennas where antenna partitioning is used. In this case, the photonic controller can consist of two subsystems, one to give the time delays required for the within-subarray antenna elements, and the other to provide the required bias delays from one subarray to another. ${ }^{23}$ The within-subarray time-delay subsystem can be implemented using the single-channel wavelength-dependent PDL module, while a larger wavelength-independent system can be used for the bias delay lines. ${ }^{24}$

\section{Switching Speed of a Ferroelectric Liquid Crystal}

In the previous sections we demonstrated two compact PDL modules based on two different FO-collimator technologies. Optical isolation of $\approx 40 \mathrm{~dB}$ and optical insertion losses of $<7 \mathrm{~dB}$ were obtained. These insertion losses are much better than the projected numbers for a compact inertialess switching PDL based on integrated electro-optic switches. We have also shown that they can be further reduced by proper design, and by eliminating the FO connectors with fiber splices. The optical isolation of our PDL is $20 \mathrm{~dB}$ better than for the integrated electro-optic approach, but not as good as for an electromechanical switch. Nevertheless, electromechanical switches are rather slow (e.g., $>7 \mathrm{~ms}) .{ }^{13}$ Thus a challenge is to obtain high switching speeds at $1319 \mathrm{~nm}$ for our FLC-based PDL modules. Figure 11 shows the switching response of our FLC devices. A 9.84- $\mu$ s rise time and a $10-\mu$ s fall time are observed. Here we define the rise time (fall time) as the time it takes for the signal to rise (fall) from the $10 \%(90 \%)$ to $90 \%(10 \%)$ of the maximum signal power. The FLC devices used were fabricated by Displaytech Inc., Longmont, $\mathrm{CO}$, and consist of three cells stacked together. This is because the FLC material used at Displaytech does not have enough birefringence for the 1319-nm band, and the preparation of a thick enough single cell using this material is at present not feasible.

Thus, the FLC-based compact PDL gives a trade-off between switching speed, optical isolation, and loss. In general, our approach gives better overall performance, as it combines the fast response time $(10 \mu \mathrm{s})$ and high optical isolation (e.g., $40 \mathrm{~dB}$ ) required for phased array antenna applications.

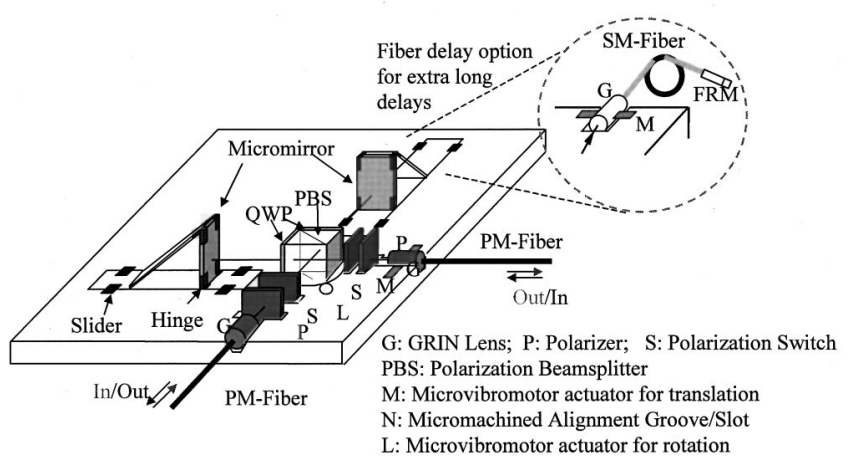

Fig. 12 An ultracompact PDL module based on MEMS technology. Both ultrashort and extra-long time-delay options are shown.

\section{Alternative Compact PDL Module Based on Microelectromechanical System Technology}

In the previous sections, we described compact PDL designs based on bulk micro-optic technology. In this section we propose an alternative approach for the implementation of ultracompact PDL modules based on the microelectromechanical system (MEMS) technology. The optical elements can be positioned on top of a silicon surfacemicromachined optical bench. Such a mechanical structure has the functionality, stability, and accuracy required for active micropositioning control of the optical elements via electromechanical microvibromotor actuators. ${ }^{25}$ This technology can lead to modules of very small size (e.g., 2.5 $\times 1 \times 0.5 \mathrm{~cm})$ and light weight (e.g., $\leqslant 0.3 \mathrm{lb}$ ).

Figure 12 shows a possible design for an ultracompact PDL. The micromirrors can be moved along the direction of the optical beams, and thus a relative time delay between the two optical paths can be obtained. An alternative option is also shown in Fig. 12, where a GRIN-lens FO collimator connectorized to a SMF is used when extra-long time delays are desirable.

\section{Conclusion}

In conclusion, we have proposed and experimentally demonstrated, for the first time, single-microbench fiberconnectorized compact PDL modules using sphericalmicrolens and GRIN-lens FO collimators, 3-D polarization optics, and FLC devices. Insertion losses of $<7 \mathrm{~dB}$ were measured for both PDL modules. Important GRIN-toGRIN coupling-loss data are presented. An approach using specially designed non-quarter-pitch FO-collimators is suggested for higher GRIN-to-GRIN coupling efficiency for the designed distances in our PDL, which can lead to further insertion-loss improvement. A low-frequency technique was used to make time-delay measurements at high RFs, thus not requiring costly microwave test equipment.

Future work relates to further PDL miniaturization and multiwavelength multichannel tests using the proposed single-physical-channel wavelength-dependent PDL. Other work to be conducted includes implementations using micromachined silicon optical benches based on MEMS technology. 


\section{References}

1. N. A. Riza, Ed., Selected Papers on Photonic Control Systems for Phased Array Antennas, SPIE Milestone Series, MS 136 (1997).

2. N. A. Riza, "Multichannel variable optical control systems for large coherent optical arrays,', in Laser Radar Technology and Application, G. W. Kamerman, Ed., Proc. SPIE 2748, 333-339 (1996).

3. N. A. Riza, "Photonically controlled ultrasonic arrays: scenarios and systems," in IEEE Ultrasonics Symp. Proc., M. Levy, S. C. Scheider, and B. R. McAvoy, Eds., Vol. 2, pp. 1545-1550 (1996).

4. J. Fu, M. Schanschula, and H. J. Caulfield, "Modular solid optic time delay systems,"' Opt. Commun. 121, 8-12 (1995).

5. N. A. Riza, "Advanced novel photonic instrumentation for adaptive and interferometric astronomy,' in Space Telescopes and Instruments $I V$, P. Y. Bely and J. B. Breckinridge, Eds., Proc. SPIE 2807, 335342 (1996).

6. N. A. Riza, 'Liquid crystal-based optical time delay units for phased array antennas,' J. Lightwave Technol. 12(8), 1440-1447 (1994).

7. N. Madamopoulos and N. A. Riza, "Directly modulated semiconductor laser fed photonic delay line using ferroelectric liquid crystals,', Appl. Opt. 37(8), 1407-1416 (1998).

8. N. A. Riza and N. Madamopoulos, "Synchronous amplitude and time delay control for an optimum dynamic range variable photonic delay line,' Appl. Opt. 38(11), 2309-2318 (1999).

9. N. A. Riza, "25-channel nematic liquid crystal optical time-delay unit characterization," IEEE Photonics Technol. Lett. 7(11), 1285-1287 (1995).

10. N. Madamopoulos and N. A. Riza, "First 7-bit true time delay photonic controller for a phased array radar," presented at OSA Annual Mtg., Symp. on RF Photonics, 1998, Baltimore, paper FU4; and N. Madamopoulos and N. A. Riza, "Demonstration of an all-digital 7-bit 33-channel photonic delay line for phased-array radars,' Appl. Opt. 39(23), (2000).

11. N. A. Riza and N. Madamopoulos, "Single micro-optical bench fiberconnectorized delay module using bulk polarization optics,' presented at Eighth Annual DARPA Symp. on Photonic Systems for Antenna Applications (PSAA-8), January 13-15, 1998, The Naval Postgraduate School, Monterey, CA, postdeadline paper.

12. N. A. Riza and N. Madamopoulos, "Reversible fiber-optic switched delay module using GRIN lens fiber-optic collimators and ferroelectric liquid crystals,' in LEOS '98 Conf. Proc.: 11th Annual Mtg. ThFF4, IEEE Lasers and Electro-Optics Soc., Orlando, FL (1998).

13. JDS Fitel Fiber Optic Catalog, JDS Fitel, Ontario, Canada.

14. UTP Catalog of Integrated Optical Circuits, UTP, Bloomfield, CT.

15. N. A. Riza and N. Madamopoulos, "Characterization of a FLC-based time delay unit for phased array antennas," J. Lightwave Technol. 15(7), 1088-1094 (1997).

16. N. A. Riza and N. Madamopoulos, "High signal-to-noise ratio birefringence compensated optical delay line based on a noise reduction scheme,' Opt. Lett. 20(22), 2351-2353 (1995).

17. Fiber Bench Coupling Systems, Optics for Research (OFR), Caldwell, NJ (1997).

18. N. A. Riza and N. Madamopoulos, "All-fiber connectorized fiberoptic delay module using 3-D polarization optics," in LEOS'97 Conf. Proc.: 10th Annual Mtg., Vol. 2, ThW2, pp. 472-473, IEEE Lasers and Electro-Optics Society, San Francisco (1997).

19. OZ-Optics Fiber Collimator/Focusers, OZ-Optics, Carp, Ontario, Canada, 1995.

20. Optics, Opto-Mechanics Catalog, OptoSigma Corp., Santa Ana, CA, 1998.

21. Garland Best, OZ-Optics, Carp, Ontario, Canada, Private Communication.

22. K. Brendin and B. Tamm, 3M Specialty Optical Fibers, West Haven, CT, Private Communications (1998).

23. A. P. Goutzoulis, D. K. Davies, J. M. Zomp, P. Hrycak, and A Johnson, "Development and field demonstration of a hardware compressive fiber-optic true time delay steering system for phased array antennas,' Appl. Opt. 33, 8173-8185 (1994).

24. N. A. Riza and N. Madamopoulos, "Phased array antenna maximum compression reversible photonic beamformer using ternary designs and multiple wavelengths,', Appl. Opt. 36(5), 983-996 (1997).

25. M. J. Daneman, N. C. Tien, O. Solgaard, A. P. Pisano, K. Y. Lau, and R. S. Muller, "Linear microvibromotor for positioning optical components,' J. Microelectromech. Syst. 5(3), 159-165 (1996).

Biographies of the authors not available. 